\title{
SIMPLY CONNECTED MANIFOLDS OF POSITIVE SCALAR CURVATURE
}

\author{
STEPHAN STOLZ
}

\begin{abstract}
Hitchin proved that if $M$ is a spin manifold with positive scalar curvature, then the $K O$-characteristic number $\alpha(M)$ vanishes. Gromov and Lawson conjectured that for a simply connected spin manifold $M$ of dimension $\geq 5$, the vanishing of $\alpha(M)$ is sufficient for the existence of a Riemannian metric on $M$ with positive scalar curvature. We prove this conjecture using techniques from stable homotopy theory.
\end{abstract}

Gromov and Lawson proved that any simply connected manifold of dimension $\geq 5$ which is nonspin admits a metric of positive scalar curvature [GL, Corollary C]. On the other hand it was shown by Lichnerowicz that not every spin manifold has a metric of positive scalar curvature [Li]. The argument is as follows: If $M$ is a $n$-dimensional spin manifold of positive scalar curvature, then by the Weizenböck formula kernel and cokernel of the Dirac operator are trivial. In particular, for $n \equiv 0 \bmod 4$ the characteristic number $\widehat{A}(M)$ which is the index of the Dirac operator vanishes. This was generalized by Hitchin, who constructed a family of Fredholm operators closely related to the Dirac operator whose index is a $K O$-characteristic number $\alpha(M) \in K O^{-n}(p t)$ [Hi, page 39]. Again using the Weizenböck formula he showed that $\alpha(M)=0$ if $M$ has a metric of positive scalar curvature. This is a strict generalization of the result of Lichnerowicz since $\alpha(M)$ can be identified with $\widehat{A}(M)$ (up to a factor) if $n \equiv 0 \bmod 4$. Gromov and Lawson proved that if $M$ is a simply connected spin manifold of dimension $\geq 5$ which is spin bordant to a manifold $N$ of positive scalar curvature, then $M$ admits a metric of positive scalar curvature [GL, Theorem B].

Received by the editors January 18, 1990 and, in revised form, June 18, 1990. 1980 Mathematics Subject Classification (1985 Revision). Primary 53C20, 55T15, 55N22, 57R90.

Key words and phrases. Positive scalar curvature, spin bordism, Adams spectral sequence.

The author is partially supported by NSF grant DHS- 88002481 . 
In this note we outline the proof of the following result which was conjectured by Gromov and Lawson.

Theorem A. Let $M$ be a simply connected spin manifold of dimension $\geq 5$. Then $M$ admits a metric with positive scalar curvature if and only if $\alpha(M)=0$.

Previously it was known that if $\alpha(M)=0$, then the connected sum $M \sharp \cdots \sharp M$ of $k$ copies of $M$ for some $k$ admits a metric of positive scalar curvature [GL, Corollary B]. Later Miyazaki proved that one can choose $k=4$ [Mi]. In dimensions $\leq 23$ Theorem A was proved by Rosenberg [Ro]. All of these results are obtained by finding explicit manifolds of positive scalar curvature representing bordism classes in the kernel of

$$
\alpha: \Omega_{n}^{\mathrm{spin}} \rightarrow K O^{-n}(p t) .
$$

The difficulties to extend this approach to prove Theorem A come from the fact that although the spin bordism groups have been computed [ABP], one does not know explicit manifolds representing a given bordism class. In particular in dimension 24 there is a bordism class for which a representative is not known.

Our proof of Theorem A is more indirect and is based on the following observation, which is well known to the experts.

Observation. Let $F$ be a compact Riemannian manifold of positive scalar curvature on which the group $G$ acts by isometries. Let $B$ be a compact manifold and let $\pi: E \rightarrow B$ be a fiber bundle with fiber $F$ and structure group $G$. Then $E$ has a metric of positive scalar curvature.

This follows from the O'Neill formulas for scalar curvature using a certain metric on $E$ with totally geodesic fibers and then shrinking the fibers [Be, 9.70d].

The strategy for the proof of Theorem A is to represent the elements of the kernel of $\alpha$ by such total spaces. The kernel of $\alpha$ is trivial in dimensions $\leq 7$ and is infinite cyclic in dimension 8 . The generator is represented by the quaternionic projective plane $\mathbf{H} P^{2}$. The standard Riemannian metric on $\mathbf{H} P^{2}$ has positive scalar curvature and so it seems natural to work with $\mathbf{H} P^{2}$ as fiber. Let $G$ be the projective symplectic group $\operatorname{PSp}(3)=\operatorname{Sp}(3) / \mathrm{Z} / 2$ and $H$ the subgroup $P(\operatorname{Sp}(2) \times \operatorname{Sp}(1))$. Given a manifold $M$ and a map $f: M \rightarrow B G$, let $\widehat{M} \rightarrow M$ be the pull back of the fiber bundle

$$
\mathbf{H} P^{2}=G / H \rightarrow B H \stackrel{\pi}{\rightarrow} B G
$$


via $f$. This bundle satisfies the assumptions of the proposition and hence $\widehat{M}$ has a positive scalar curvature metric. Moreover, if $M$ is spin, then $\widehat{M}$ is again a spin manifold. We can hence define a homomorphism

$$
\Psi: \Omega_{n}^{\mathrm{spin}}(B G) \rightarrow \Omega_{n+8}^{\mathrm{spin}}
$$

by mapping the bordism class of $f: M \rightarrow B G$ to the bordism class of $\widehat{M}$.

Theorem B. Let $[M]$ be a bordism class in the kernel of $\alpha$. Then there is an odd number $k$ such that $k[M]$ is in the image of $\Psi$.

It is possible to strengthen this result: One can always choose $k=1[\mathrm{KS}]$ and this stronger result clearly implies Theorem A. Alternatively, one can use Miyazaki's result to derive Theorem A as follows. Suppose that the bordism class of a simply connected spin manifold $M$ of dimension $\geq 5$ is in the kernel of $\alpha$. Then the connected sum $k M$ of $k$ copies of $M, k$ odd, is spin bordant to the total space of an $\mathbf{H} P^{2}$-bundle and hence carries a metric of positive scalar curvature by Theorem $B$ above and the GromovLawson result. On the other hand $4 M$ carries a metric of positive scalar curvature by Miyazaki's result. Since $k$ and 4 are relatively prime, we can find natural numbers $a, b$ such that $1=a \cdot 4-b \cdot k$. Then $M$ is spin bordant to the disjoint union of $a$ copies of $4 M$ and $b$ copies of $k \bar{M}$ where $\bar{M}$ denotes the manifold $M$ with reversed orientation. Hence $M$ admits a metric of positive scalar curvature.

Theorem B is proved by translating the statement into stable homotopy theory via the Pontrjagin-Thom construction and then using Adams spectral sequence techniques. Recall that $\Omega_{n}^{\text {spin }}$ is canonically isomorphic to $\pi_{n} M$ Spin where $M$ Spin is the Thom spectrum associated to the projection map $B$ Spin $\rightarrow B O$. More generally, for any space $X$ the bordism group $\Omega_{n}^{\text {spin }}(X)$ can be identified with $\pi_{n} M$ Spin $\wedge X_{+}$, where $X_{+}$is the union of $X$ and a disjoint basepoint. Associated to the fiber bundle $(*)$ there is a Thom map

$$
T(\pi): \Sigma^{8} B G_{+} \rightarrow M(-\tau),
$$

where $\tau$ is the tangent bundle along the fibers and $M(-\tau)$ is the Thom spectrum of $-\tau$ [Bo, 6.20]. Let $c(-\tau): E \rightarrow B$ Spin be the classifying map of $-\tau$, let $M c(-\tau)$ be the induced map of Thom spectra, let $t$ be the composition of $T(\pi)$ and $M c(-\tau)$ and let 
$T$ be the composition

$$
M \text { Spin } \wedge \Sigma^{8} B G_{+} \stackrel{i d \wedge t}{\rightarrow} M \text { Spin } \wedge M \text { Spin } \stackrel{\mu}{\rightarrow} M \text { Spin } .
$$

Here $\mu$ is the multiplication map of the ring spectrum $M$ Spin corresponding to taking products of spin manifolds. Then $\Psi$ can be identified with the induced map $T_{*}$ on homotopy groups [Bo, Theorem 6.24].

Localized at 2 the spectrum $M$ Spin splits as a wedge of smaller spectra [ABP]. In particular, it is homotopy equivalent to $b o \mathrm{~V}$ $M$ Spin, where $b o$ is the connective real $K$-theory spectrum and $M$ Spin is the wedge of the other summands. Let $p_{1}: M$ Spin $\rightarrow$ $b o$ (resp. $p_{2}: M \operatorname{Spin} \rightarrow \widetilde{M \text { Spin) }}$ be the projection maps. The map induced by $p_{1}$ on homotopy groups can be identified with the homomorphism $\alpha$. The following is then the stable homotopy version of Theorem $B$.

Theorem $\mathbf{B}^{\prime}$. Localized at 2 the map induced by $p_{2} T$ on homotopy groups is surjective.

Theorem $\mathbf{B}^{\prime}$ is proved using Adams spectral sequence techniques. Recall that the $\mathrm{Z} / p$-homology $H_{*} X$ of a spectrum $X$ is a comodule over the dual Steenrod algebra $A_{*}$. If $H_{*} X$ is known as $A_{*}$-comodule, one can use the $\bmod p$ Adams spectral sequence

$$
\mathrm{Ext}_{A_{*}}^{s, t}\left(\mathbf{Z} / p, H_{*} X\right) \Rightarrow_{p} \pi_{t-s}(X)
$$

to obtain information about the $p$-primary part ${ }_{p} \pi_{*}(X)$ of the homotopy groups of $X$ [Sw, 19.9 and 19.12]. From now on let $p=2$.

Proposition C. The induced map $\left(p_{2} T\right)_{*}: H_{*} M \operatorname{Spin} \wedge \Sigma^{8} B G_{+} \rightarrow$ $H_{*} M \widetilde{\text { Spin }}$ is a split surjection of $A_{*}$-comodules.

As a corollary we get that the induced map

$$
\begin{aligned}
\left(p_{2} T\right)_{*}: & \mathrm{Ext}_{A_{*}}^{s, t}\left(\mathbf{Z} / 2, H_{*} M \operatorname{Spin} \wedge \Sigma^{8} B G_{+}\right) \\
& \rightarrow \operatorname{Ext}_{A_{*}}^{s, t}\left(\mathbf{Z} / 2, H_{*} M \text { Spin }\right)
\end{aligned}
$$

is a surjection of the $E_{2}$-terms of the corresponding Adams spectral sequences. Note that this does not imply that $p_{2} T$ induces a surjection of the $E_{\infty}$-terms, since there could be nontrivial differentials in the domain spectral sequence. Fortunately this is not the case.

Proposition D. The mod 2 Adams spectral sequence of $M$ Spin $\wedge \Sigma^{8} B G_{+}$collapses. 
Hence $p_{2} T$ induces a surjection of the $E_{\infty}$-terms which implies Theorem $\mathrm{B}^{\prime}$. To prove Proposition $\mathrm{D}$ we show that $H_{*} M$ Spin $\wedge \Sigma^{8} B G_{+}$is isomorphic to an extended $A(1)_{*}$-comodule $A_{*} \square_{A(1)_{*}} M$, where $A(1)_{*}$ is the dual of $A(1)$, the subalgebra of the Steenrod algebra generated by $S q^{1}$ and $S q^{2}$, and $M$ is an $A(1)_{*}$-comodule. Then

$$
\mathrm{Ext}_{A_{*}}^{s, t}\left(\mathrm{Z} / 2, H_{*} M \operatorname{Spin} \wedge \Sigma^{8} B G_{+}\right) \cong \mathrm{Ext}_{A(1)_{*}}^{s, t}(\mathrm{Z} / 2, M)
$$

by [Sw, Proposition 20.16]. Moreover, $M$ is a direct sum of $A(1)_{*}$-comodules, whose Ext-groups are known [AP, §3]. Let $Y$ be the spectrum obtained from $M$ Spin $\wedge \Sigma^{8} B G_{+}$by splitting off the Eilenberg-MacLane spectrum corresponding to the free summands of $M$ [Ma]. Inspecting the $E_{2}$-term of the mod 2 Adams spectral sequence of $Y$, we conclude that all differentials are zero for dimensional reasons and due to the multiplicative structure. This implies Proposition D.

For the proof of Proposition C, we first show using results of D. Pengelley [P] that $H_{*} M \widetilde{\text { Spin }} \cong A_{*} \square_{A(1)} \bar{N}$, where $\bar{N}$ is a certain $A(1)_{*}$-comodule. Moreover, $\left(p_{2} T\right)_{*}$ is induced by a map of $A(1)_{*}$ comodules $f: M \rightarrow \bar{N}$. A calculation shows that $f$ is surjective and that $f$ induces a surjection on the $Q_{0}$-homology groups $\left(Q_{0}\right.$ is the Bockstein which acts as a differential on $A(1)_{*}$-comodules). It turns out that this suffices to conclude that $f$ is a split surjection of $A(1)_{*}$-comodules which implies Proposition C.

\section{REFERENCES}

[AP] J. F. Adams and S. Priddy, Uniqueness of BSO, Math. Proc. Cambridge Philos. Soc. 80 (1978), 475-509.

[ABP] D. W. Anderson, E. H. Brown, Jr., and F. P. Peterson, The structure of the spin cobordism ring, Ann. of Math. 86 (1967), 271-298.

[Be] A. L. Besse, Einstein manifolds, Springer-Verlag, Berlin and New York, 1986.

[Bo] J. M. Boardman, Stable homotopy theory; Chapter V-Duality and Thom spectra, mimeographed notes, Warwick, 1966.

[GL] M. Gromov and H. B. Lawson, Jr., The classification of simply connected manifolds of positive scalar curvature, Ann. of Math. 111 (1980), 423-434.

[Hi] N. Hitchin, Harmonic Spinors, Adv. Math. 14 (1974), 1-55.

[KS] M. Kreck and S. Stolz, A geometric interpretation of elliptic homology (in preparation).

[Li] A. Lichnerowicz, Spineurs harmoniques, C. R. Acad. Sci. Paris Sér. A-B 257 (1963), 7-9. 
[Ma] H. R. Margolis, Eilenberg-MacLane spectra, Proc. Amer. Math. Soc. 43 (1974), 409-415.

[Mi] T. Miyazaki, Simply connected spin manifolds and positive scalar curvature, Proc. Amer. Math. Soc. 93 (1985), 730-734.

[P] D. J. Pengelley, $H^{*}(M O\langle 8\rangle ; \mathrm{Z} / 2)$ is an extended $A_{2}^{*}$-coalgebra, Proc. Amer. Math. Soc. 87 (1983), 355-356.

[Ro] J. Rosenberg, $C^{*}$-algebras, positive scalar curvature, and the Novikov Conjecture, III, Topology 25 (1986), 319-336.

[Sw] R. M. Switzer, Algebraic topology-homotopy and homology, SpringerVerlag, Berlin and New York, 1975.

Department of Mathematics, University of Notre Dame, Notre Dame, INDIANA 46556

E-mail address: stolz@iRIsHMvs.BitNeT 